\title{
Penetrating Abdominal Trauma in Children: A Tertiary Hospital Experience in a Developing Country
}

Chukwubuike Kevin Emeka

Pediatric Surgery Unit, Department of Surgery, Enugu State University Teaching Hospital, Enugu, Nigeria.

*Corresponding author: Chukwubuike Kevin Emeka, Pediatric Surgery Unit, Department of Surgery, Enugu State University Teaching Hospital, Enugu, Nigeria.

Received date: May 05, 2021; Accepted date: May 11, 2021; Published date: May 19, 2021

Citation: Chukwubuike K Emeka (2021). Penetrating Abdominal Trauma in Children: A Tertiary Hospital Experience in a Developing Country. $J$ Clinical Research and Reports, 8(1); DOI:10.31579/2690-1919/168

Copyright: (C) 2021 Chukwubuike Kevin Emeka, This is an open access article distributed under the Creative Commons Attribution License, which permits unrestricted use, distribution, and reproduction in any medium, provided the original work is properly cited.

\begin{abstract}
Objective: Penetrating abdominal trauma in children is a life threatening emergency. The objective of this study was to evaluate our experience in the management of penetrating abdominal trauma in children in a tertiary hospital in Enugu, Nigeria.

Materials and Methods: This was a retrospective study of children that were managed for penetrating abdominal injury at the pediatric surgery unit of Enugu State University Teaching Hospital, Enugu, Nigeria. Medical records of the patients over a 10-year period were evaluated.

Results: During the study period, there were 21 cases of penetrating abdominal injuries with an age range of 9 to 15 years (median: 11 years) and male to female ratio of 4.25:1. Fourteen $(66.7 \%)$ patients presented after 48 hours from the time of the incident. Gunshot injury was the most common mechanism of injury. Five (23.8\%) patients were in a clinical state of shock on presentation to the hospital and $10(47.6 \%)$ patients had evisceration of intra-abdominal organ. Small intestine was the most damaged intra-abdominal organ while head injury was the most associated extraabdominal injury. Surgical site infection was a common post-operative complication and 2 (9.5\%) patients expired.
\end{abstract}

Conclusion: Penetrating abdominal trauma in children is uncommon. Late presentation is common in low income countries and the small intestine is the most damaged intra-abdominal organ.

Keywords: abdominal; children; penetrating; trauma; tertiary hospital; laparotomy; howbeit

\section{Introduction}

Trauma is a physical injury caused by transfer of energy to and within the person involved and is a major cause of morbidity and mortality in children globally [1]. The abdomen is the third most injured part of the body after the head and extremities: Howbeit, the most unrecognizable fatal injuries missed on initial assessment occur in the abdomen [2]. Most abdominal injuries are blunt in nature but penetrating abdominal injuries from stab wound, gunshots and impalement injuries can occur. Other causes of penetrating injuries include fall onto sharp objects, road traffic accidents, injuries by animal horns and sporting injuries [3]. Penetrating abdominal injury is usually accidental and unintentional in children and is less common in children than in adults [4]. Insurgency attacks, civil and communal conflicts have increased the incidence of penetrating abdominal trauma. The absence of plausible mechanism of injury by the guardian may suggest child abuse [2]. Penetrating abdominal trauma is an acute life threatening emergency [5]. Radiological evaluation of children who sustain penetrating abdominal injury involves the use of ultrasound and computed tomography scan. Ultrasound has sensitivity of $56-97 \%$ in detecting intra-abdominal injuries. Computed tomography scan is the gold standard in the diagnosis of abdominal injuries [6]. The approach to the management of penetrating abdominal injuries depends on the type of object that caused the injury and hemodynamic status. For instance, gunshot to the abdomen is usually associated with hollow viscus injury and mostly requires laparotomy [7].

\section{Materials and Methods}

This was a retrospective study of children aged 15 years and below who sustained penetrating abdominal injury and were managed at the pediatric surgery unit of Enugu State University Teaching Hospital (ESUTH) Enugu, Nigeria between January 2007 and December 2017. All pediatric patients with penetrating abdominal injuries seen during the study period were included in the study. Patients with incomplete case records and those older than 15 years of age were excluded. Penetrating anal injuries were also excluded. ESUTH is a tertiary hospital located in Enugu, South East Nigeria. The hospital serves the whole of Enugu State, which according to the 2016 estimates of the National Population Commission and Nigerian National Bureau of Statistics, has a population of about 4 million people and a population density of $616.0 / \mathrm{km}^{2}$. The hospital also receives referrals from its neighboring states. Information was obtained from the case notes, operation notes, operation register, and admissiondischarge records. The information obtained included the age, gender, 
interval between the incident and presentation, mechanism of injury, hemodynamic status at presentation, presence/absence of abdominal organ evisceration, associated extra-abdominal injuries, intra-operative finding, operative procedure performed, complications of treatment, duration of hospital stay and outcome of treatment. The period of follow up was for 12 months.

Ethical approval was obtained from the ethics and research committee of ESUTH. This research conforms to the principles of the Declaration of Helsinki. The author certifies that they have obtained patient consent forms. In the form the patients have given their consent for their data to be reported in a journal. The patients understand that their names will not be published and due efforts will be made to conceal their identity. Statistical Package for Social Science (SPSS) for Windows version 23 (IBM Corp., Armonk, NY) was used for data entry and analysis. Data were expressed as percentages, median, mean, and range.

\section{Pre-operative protocol}

On presentation to the hospital, the patients were clinically evaluated and resuscitated using Advanced Trauma Life Support (ATLS) protocol. Urgent hemoglobin estimation was done. Anemia, if present, was corrected and the patient taken to theatre for surgery. Abdominal $\mathrm{x}$ ray and ultrasound were performed in patients who do not have bowel evisceration. Those with bowel evisceration were taken straight to theatre. All the patients had laparotomy.

\section{Intra-operative protocol}

Abdominal access was through transverse supraumbilical incision. An exploration was performed and any damaged hollow viscus or solid organ was repaired as appropriate. In a background of gross peritoneal contamination, a temporary enterostomy was performed. All surgical procedures were performed by a consultant pediatric surgeon under general anesthesia.

\section{Post-operative protocol}

The patients were placed on parenteral antibiotics and analgesics. Graded oral sips were commenced when bowel function returned. Post-operative complications were noted and documented. Discharge was based on recovery from surgery, full establishment of oral intake, ambulation and adequate wound healing.

\section{Results}

\section{Patients' demographics}

A total of 108 cases of abdominal traumas were recorded in children during the study period out of which 21 cases were penetrating abdominal injuries. Penetrating abdominal injuries accounted for $19.4 \%$ of all abdominal injuries. The peak age group of the patients was 10-14 years. Details of the demographics are shown in Table 1.

\begin{tabular}{|cc|}
\hline \multicolumn{1}{|c|}{ Mender } & $17(81 \%)$ \\
Female & $4(19 \%)$ \\
Median age of the patients & 11 years $(9-15)$ \\
Median interval between incident and presentation & 2 days $(1-3)$ \\
Presented within 24 hours & $3(14.3 \%)$ \\
Presented between 24 and 48 hours & $4(19 \%)$ \\
Presented after 48 hours & $14(66.7 \%)$ \\
The mean duration of hospital stay & 14 days $(10-20)$ \\
\hline
\end{tabular}

Table 1. Demographic profile of the patients

\section{Mechanism of injury}

The mechanisms of injury are shown in Table 2.

\begin{tabular}{|c|c|c|}
\hline Mechanism of injury & Number & Percentage \\
\hline Gunshot & 9 & 42.9 \\
\hline Fall & 6 & 28.6 \\
\hline Impalement & 3 & 14.3 \\
\hline Road traffic accident & 2 & 9.5 \\
\hline Animal horn & 1 & 4.7 \\
\hline
\end{tabular}

Table 2. Mechanism of injury

\section{Hemodynamic status at presentation}

Five $(23.8 \%)$ patients were in a clinical state of shock on presentation to the hospital. Clinical shock was characterized by hypotension, narrow pulse pressure, tachycardia and oliguria.

\section{Abdominal organ evisceration at presentation}

Ten $(47.6 \%)$ patients had evisceration of the intra-abdominal organ. Details of the eviscerated organs are shown in Table 3.

\begin{tabular}{|c|c|c|}
\hline Organ & Number & Percentage \\
\hline Omentum & 6 & 28.6 \\
\hline Intestine & 3 & 14.3 \\
\hline Stomach & 1 & 4.8 \\
\hline
\end{tabular}

Table 3. Eviscerated organs

\section{Associated extra-abdominal injuries}

There was no associated injury in $16(76.2 \%)$ patients and $5(23.8 \%)$ had associated injuries. Details are depicted in Figure 1. 


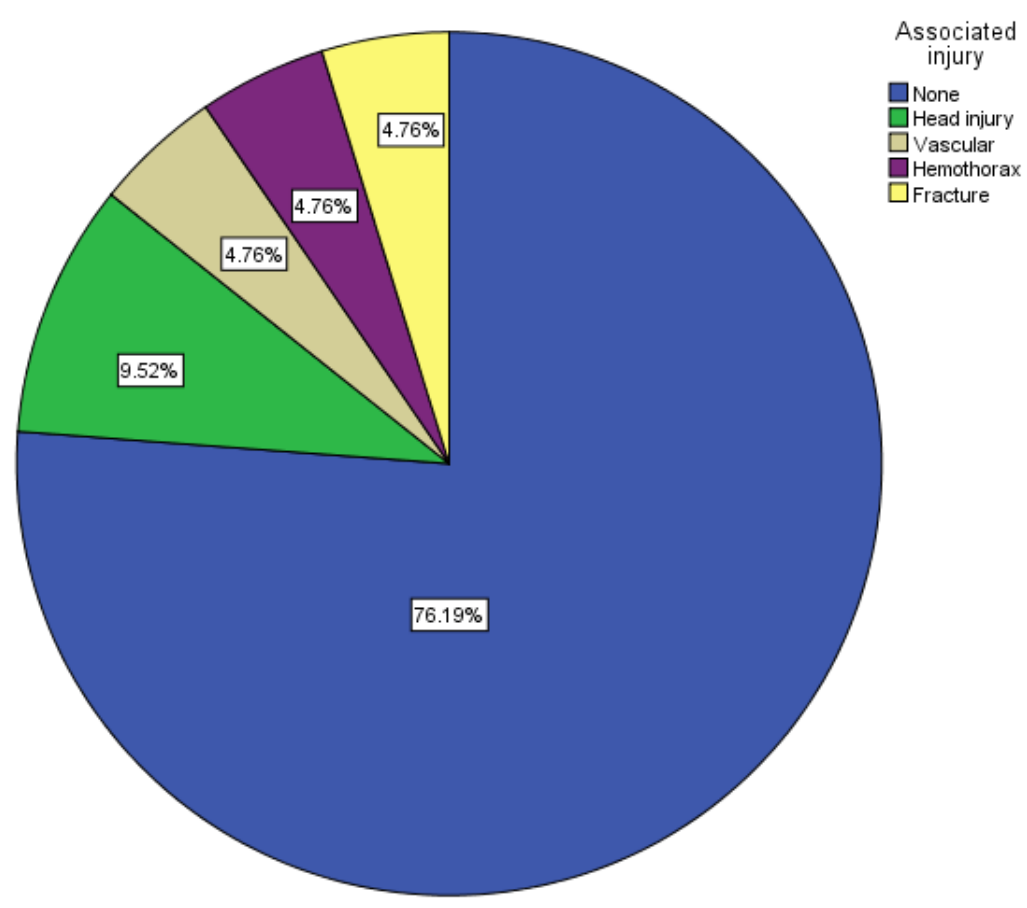

Figure 1. Associated injuries

3.6. Intra-operative finding and surgical procedure performed

Injuries to the intra-abdominal organs occur in various combinations. The injured organs and the procedures performed are shown in Table 4.

\begin{tabular}{|c|c|c|}
\hline Damaged organ & Number of patients (\%) & Procedure performed \\
\hline Perforated small intestine & $13(61.9)$ & Repair $10(47.6 \%)$ \\
\hline & & RHC with ITA 3 (14.3\%) \\
\hline Perforated large intestine & $8(38)$ & Colostomy \\
\hline Stomach perforation & $6(28.6)$ & Repair \\
\hline Liver injuries & $6(28.6)$ & Debridement and repair \\
\hline Splenic injuries & $4(19)$ & Repair \\
\hline Urinary bladder injuries & $2(9.5)$ & Repair \\
\hline Vascular injury & $1(4.8)$ & Repair \\
\hline
\end{tabular}

RHC=Right hemicolectomy; ITA=Ileotransverse anastomosis

Table 4. Operative finding and operation performed

\section{Post-operative complications}

Fourteen (66.7\%) patients did not develop any complications. Five (23.8\%) patients had surgical site infection, one (4.8\%) each had intraabdominal abscess and burst abdomen.

\section{Outcome of treatment}

Nineteen $(90.5 \%)$ patients recovered and were discharged home. Mortality was recorded in $2(9.5 \%)$ patients.

\section{Discussion}

Penetrating abdominal trauma typically involves the violation of the abdominal cavity by an object. Some trauma scores such as Penetrating Abdominal Trauma Index (PATI) and Injury Severity Score (ISS) have been used to assess the severity and relative risk of morbidity and mortality associated with penetrating abdominal trauma [8]. Penetrating abdominal injuries have traditionally being managed by routine laparotomy. However, non-operative management can be applied to appropriate cases [9]. The practice of deciding which patients will not need operation following penetrating abdominal trauma is termed selective management [10].

In the present study, penetrating abdominal injury accounted for about one-fifth of all abdominal injuries. This finding is inconsistent with the report from northern Nigeria where there is a higher incidence of penetrating abdominal trauma [3]. The reason for the higher incidence of penetrating abdominal injuries in northern Nigeria could be due to increased communal conflicts and Boko Haram insurgency in that part of the country. Peak age group in our patients is comparable to the reports of other researchers $[2,11]$. But is at variance with the report of others [12]. The peak age affected in penetrating abdominal injury may depend on the predominant mechanism of injury. For instance, older children are more likely to be affected by gunshot and stab injuries while toddlers may be more involved in falls at home. The male predominance observed in the current study is consistently reported by other authors $[12,13]$. This male predominance may be due to involvement of male children in assisting their families in farming and herding in low income countries [13]. Late presentation of our patients is evidenced in the median 2 days 
waiting time before presentation to the hospital. A study done in Maiduguri, Nigeria also reported delayed presentation of the patients with penetrating abdominal injury: One of their study patients presented after 6 days from the date of the penetrating abdominal trauma [12]. This late presentation may be due to poverty and ignorance that is prevalent in developing countries. The average length of time the patients stayed in the hospital in the current study is comparable to the reports of Chalya et al but unsupported by the works of De Robles [14, 15]. The duration of time a patient stays in the hospital may depend on the age of the patient, severity of injury and extent of the surgical procedure performed [16].

Penetrating abdominal injury by gunshot was the most frequent mechanism of injury in the present study. The gunshot injuries resulted from communal clashes and accidental discharges while playing with their fathers' firearms. Wabata et al also reported gunshot as a common cause of penetrating abdominal injury in children [12]. However, Ameh et al reported fall onto sharp objects within and around the home as the most common cause of penetrating abdominal injury in children [3]. In areas of terrorist attacks and civil war, incidence of abdominal gunshot injuries is high $[17,18]$. Penetrating abdominal injuries in children that occur at home usually result from accidental falls [19].

About one quarter of our patients were in shock at presentation. One study reported that more than $50 \%$ of their patients were hemodymically unstable as at the time of hospital admission [12]. Hemodynamic instability following penetrating abdominal injury may result from hypovolemia due to blood loss or from sepsis in hollow viscus perforation in late presenters.

Unlike in blunt abdominal trauma, penetrating abdominal injury may be associated with evisceration of intra-abdominal organ. Omentum was the most common eviscerated structure in the present study. This is comparable to the report of other studies $[12,20]$. However, there are reports of the intestine and other organs eviscerating in penetrating abdominal injury $[19,21]$. The organ eviscerated in penetrating abdominal trauma may depend on the cause of the penetrating abdominal trauma and extent of damage to the abdominal wall.

Extra-abdominal injuries may be associated with penetrating abdominal trauma. In line with our finding, a study from Tanzania also reported head injury as the most associated extra-abdominal injury [14]. However, fractures of long bones could be the most associated injury [22]. The pattern and mechanism of injury may determine the possible associated injuries.

The small intestine was the most common injured organ in the present study. Other studies also reported small intestine as the most injured organ $[2,7]$. Other intra-abdominal organs may also be damaged in penetrating abdominal trauma [11]. Surgical site infection was the most common post-operative complication recorded in the present study. Other studies also reported surgical site infection as a common complication following laparotomy for penetrating abdominal [23, 24]. Complications such as enterocutanous fistula can occur when there is anastomotic leak. The mortality rate in the present study is comparable to a similar study carried out in Maiduguri, Nigeria [12]. Mortality from the current study was from overwhelming sepsis due to infectious complications. Dellinger et al in their series reported that following laparotomy for penetrating abdominal injury, 9 out of 10 deaths were directly related to infectious complications [25].

\section{Conclusion:}

Although penetrating abdominal trauma in children is far less common than blunt abdominal trauma, its treatment is surgical and could be associated with some mortality. Late presentation is common in low income countries and small intestine was the most injured intra- abdominal organ. Prevention is important as most of the injuries can be avoided through effective parenteral control and resolution of conflicts.

\section{Declarations}

Acknowledgement: My gratitude goes to the resident doctors who helped in data collection.

\section{Conflict of interest: None}

\section{Funding: None}

Ethical considerations: Ethical approval was obtained from ethics and research committee of the hospital.

\section{References}

1. Haagsma JA, Graetz N, Bolliger I, Naghavi M, Higashi H, Mullany EC. (2013) The global burden of injury: Incidence, mortality, disability-adjusted life years and time trends from the Global Burden of Disease study. Inj Prev; 22(1): 3-18.

2. Sinha CK, Lander A. (2013) Trauma in children: abdomen and thorax. Surgery (oxford); 31(3): 123-129.

3. Ameh EA, Nmadu PT. (1999) Penetrating abdominal injuries in children in Nigeria. Ann Trop Paediatr; 19(3): 293-296.

4. Nwokoro CC, Emmanuel EA, Fatungase OM, Salami BA, Ogundele IO, Amosu LO. (2017) Impalement abdominal injury in a 6-year-old child. Afr J Med Health Sci: 16: 126-128.

5. Mikrogianakis A. (2010) Penetrating Abdominal Trauma in Children. Clinical Pediatric Emergency Medicine; 11(3): 217224.

6. Gaines BA. (2009) Intra-abdominal solid organ injury in children: diagnosis and treatment. J Trauma; 67(2 Suppl): S135-S139.

7. Lotfollahzadeh S, Burns B. (2020) Penetrating Abdominal Trauma. In: Treasure Island (FL). StatPearls Publishing.

8. Dogjani A, Petrela E, Jonuzaj E, Blenarda H, Najada K. (2016) PATI Index \& Injury Severity Score, as predictive factors in management of Penetrating abdominal Trauma. Eur J Trauma Emerg Surg; 42 (Suppl 2): 9-S245.

9. Butt MU, Zacharias N, Velmahos GC. (2009) Penetrating abdominal injuries: management controversies. Scand J Trauma Resusc Emerg Med; 17: 19.

10. Como JJ, Bokhari F, Chiu WC, Duane TM, Holevar MR, Tandoh MA et al. (2010) Practice management guidelines for selective non-operative management of penetrating abdominal trauma. J Trauma; 68(3): 721-733.

11. Sheshe AA, Yakubu AA. (2017) Analysis of pattern and outcome of abdominal trauma in a tertiary hospital in Kano, Northwestern Nigeria. Arch Int Surg; 7: 22-26.

12. Wabada S, Abubakar AM, Chinda JY, Adamu S, Bwala KJ. (2018) Penetrating abdominal injuries in children. Annals of Pediatric Surgery; 14(1): 8-12.

13. Hargrave JM, Pearce P, Mayhew ER, Bull Anthony, Taylor S. (2019) Blast injuries in children: a mixed-methods narrative review. BMJ Paediatrics Open; 3: e000452.

14. Chalya PL, Mabula JB. (2013) Abdominal trauma experience over a two-year period at a tertiary hospital in north-western Tanzania: a prospective review of 396 cases. Tanzania Journal of Health Research; 15(4).

15. De Robles MB, Ayuste Jr EC. (2019) Mandatory Laparotomy in Penetrating Abdominal Injuries with Omental Eviscerations: Experience in a Major Trauma Center in the Philipines. Cureus; 11(9): e5688.

16. Haghparast-Bidgoli H, Saadat S, Bogg L, Yarmohammadian MH, Hasselberg M. (2013) Factors affecting hospital length of stay and hospital charges associated with road traffic-related injuries in Iran. BMC Health Serv Res; 13: 281. 
17. Ojo EO, Ozoilo KN, Sule AZ, Ugwu BT, Misauno MA, Ismaila BO, Peter SD et al. (2016) Abdominal injuries in communal crises: The Jos experience. J Emerg Trauma Shock; 9: 3-9

18. Chianakwana GU, Mbonu OO, Egwuonwu AO, Azike J, Eleweke N, Ekwunife C et al. (2017) Missile and blast injuries in Nigeria: Southeast experience. J West Afr Coll Surg; 7(4): 18-33

19. Abdulraheem NT, Osuoji RI, Akanbi OR, Abdur-Rahman LO, Nasir AA. (2015) A rare form of domestic accidents: Penetrating abdominal injuries with evisceration of abdominal viscus. Afr $\mathbf{J}$ Trauma; 4: 30-33.

20. Yucel M, Ozpek A, Yuksekdag S, Kabak I, Basak F, Kilik A et al. (2014) The management of penetrating stab wounds with organ or omental evisceration. The results of a clinical trial. Ulus Cerrahi Derg; 30(4): 207-210.
21. Riley P. (1987) Disembowelment-a retrospective study of patients suffering evisceration following penetrating abdominal injury. Injury; 18(4): 286-290.

22. Sude NS, Suryawanshi PR. (2014) Blunt Abdominal Trauma in RTA Patient-A State of Industrial Anarchy. International Journal of Science and Research; 3(10):806-812.

23. Ohene -Yeboah M, Dakubo JC, Boakye F, Naeeder SB. (2010) Penetrating abdominal injuries in adults seen at two teaching hospitals in Ghana. Ghana Med J; 44(3): 103-108.

24. Olagun JG, Etonyeaku AC, Ige JT, Wuraola OK. (2020) Penetrating abdominal injuries in an emerging semi-urban teaching hospital; 6(1): 7-12.

25. Dellinger EP, Oreskovich MR, Wertz MJ, Hamasaki V, Lennard ES. (1984) Risk of infection following laparotomy for penetrating abdominal injury. Arch Surg; 119(1): 20-27.

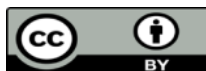

This work is licensed under Creative Commons Attribution 4.0 License

To Submit Your Article Click Here: Submit Manuscript

DOI: $10.31579 / 2690-1919 / 168$

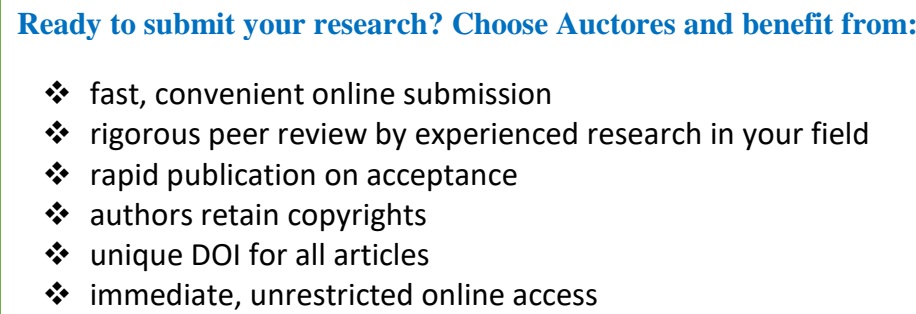

At Auctores, research is always in progress.

Learn more www.auctoresonline.org/journals/journal-of-clinicalresearch-and-reports- 